Development of a Primary Care Guide for Implementing Evidence-Based Screening and Counseling for Unhealthy Alcohol Use With EpicBased Electronic Health Record Tools: A Pilot Dissemination Project

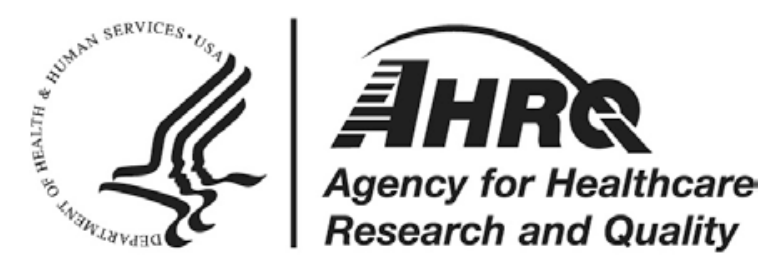




\section{Development of a Primary Care Guide for Implementing Evidence-Based Screening and Counseling for Unhealthy Alcohol Use With Epic- Based Electronic Health Record Tools: A Pilot Dissemination Project}

Prepared for:

Agency for Healthcare Research and Quality

U.S. Department of Health and Human Services

5600 Fishers Lane

Rockville, MD 20857

www.ahrq.gov

Contract No. 290-2015-00011-I, Task Order 1

Prepared by:

RTI International-University of North Carolina Evidence-based Practice Center

Research Triangle Park, NC

Investigators:

Colleen Barclay, M.P.H.

Meera Viswanathan, Ph.D.

Daniel E. Jonas, M.D., M.P.H.

AHRQ Publication No. 18-EHC020-EF

September 2018 


\section{Key Messages}

- Findings: We evaluated the initial implementation of an evidence-based, systematic approach to screening and counseling for unhealthy alcohol use in a General Internal Medicine (GIM) Clinic that included Epic electronic health record (EHR)tools. Factors facilitating implementation included leadership support and organizational culture; a multidisciplinary team; and staff and provider buy-in. EHR tools increased service provision but could contribute to alert fatigue. Competing demands, a large patient population, and turnover in nursing staff and resident providers were identified as potential barriers. We then developed a dissemination package intended to offer a practical roadmap to the process of integrating these evidence-based services into a clinic's workflow. Key Informants (representatives of health systems in a position to make decisions about implementation of services into primary care) viewed the package very favorably.

- Lessons Learned for EPC Program: Developing dissemination packages based on Evidence-based Practice Center reports would require expansion of the timeline and resources. Creation of support tools would be facilitated by working with a team that has recently implemented the clinical service in question. Development of the dissemination package for the current pilot project was preceded by nearly 2 years in which we worked on implementation in the clinical setting.

- Utility for Health Systems: Key Informants reviewed a draft of the package and completed a questionnaire about its usefulness. They found package components providing direct, practical guidance for implementation to be very useful; components dealing with more general aspects of implementation were also rated as very useful by most but as somewhat useful or not particularly useful by some. 
This report is based on research conducted by the RTI International-University of North Carolina Evidence-based Practice Center (EPC) under contract to the Agency for Healthcare Research and Quality (AHRQ), Rockville, MD (Contract No. 290-2015-00011-I). The findings and conclusions in this document are those of the authors, who are responsible for its contents; the findings and conclusions do not necessarily represent the views of AHRQ. Therefore, no statement in this report should be construed as an official position of AHRQ or of the U.S. Department of Health and Human Services.

Colleen Barclay and Daniel Jonas received funding from the Institute for Healthcare Quality Improvement, University of North Carolina at Chapel Hill, for the implementation/quality improvement project on which this pilot project was based.

None of the investigators have any affiliations or financial involvement that conflicts with the material presented in this report.

The information in this report is intended to help health care decisionmakers-patients and clinicians, health system leaders, and policymakers, among others-make well-informed decisions and thereby improve the quality of health care services. This report is not intended to be a substitute for the application of clinical judgment. Anyone who makes decisions concerning the provision of clinical care should consider this report in the same way as any medical reference and in conjunction with all other pertinent information, i.e., in the context of available resources and circumstances presented by individual patients.

This report is made available to the public under the terms of a licensing agreement between the author and the Agency for Healthcare Research and Quality. This report may be used and reprinted without permission except those copyrighted materials that are clearly noted in the report. Further reproduction of those copyrighted materials is prohibited without the express permission of copyright holders.

AHRQ or U.S. Department of Health and Human Services endorsement of any derivative products that may be developed from this report, such as clinical practice guidelines, other quality enhancement tools, or reimbursement or coverage policies may not be stated or implied.

Persons using assistive technology may not be able to fully access information in this report. For assistance contact EPC@ahrq.hhs.gov.

Suggested citation: Barclay C, Viswanathan M, Jonas DE. Development of a Primary Care Guide for Implementing Evidence-based Screening and Counseling for Unhealthy Alcohol Use with Epic-based Electronic Health Record Tools: A Pilot Dissemination Project. Methods Research Report. (Prepared by RTI International-University of North Carolina Evidence-based Practice Center under Contract No. 290-2015-00011-I). AHRQ Publication No. 18-EHC020-EF. Rockville, MD: Agency for Healthcare Research and Quality; September 2018. Posted final reports are located on the Effective Health Care Program search page. DOI: https://doi.org/10.23970/AHRQEPCMETHENGAGEALCOHOL. 


\section{Preface}

The Agency for Healthcare Research and Quality (AHRQ), through its Evidence-based Practice Centers (EPCs), sponsors the development of evidence reports and technology assessments to assist public- and private-sector organizations in their efforts to improve the quality of health care in the United States. The reports and assessments provide organizations with comprehensive, science-based information on common, costly medical conditions and new health care technologies and strategies. The EPCs systematically review the relevant scientific literature on topics assigned to them by AHRQ and conduct additional analyses when appropriate prior to developing their reports and assessments.

To improve the scientific rigor of these evidence reports, AHRQ supports empiric research by the EPCs to help understand or improve complex methodologic issues in systematic reviews. These methods research projects are intended to contribute to the research base in and be used to improve the science of systematic reviews. They are not intended to be guidance to the EPC program, although may be considered by EPCs along with other scientific research when determining EPC program methods guidance.

AHRQ expects that the EPC evidence reports and technology assessments will inform individual health plans, providers, and purchasers as well as the health care system as a whole by providing important information to help improve health care quality. The reports undergo peer review prior to their release as a final report.

If you have comments on this Methods Research Project they may be sent by mail to the Task Order Officer named below at: Agency for Healthcare Research and Quality, 5600 Fishers Lane, Rockville, MD 20857, or by email to epc@ahrq.hhs.gov.

Gopal Khanna, M.B.A.

Director

Agency for Healthcare Research and Quality

Stephanie Chang, M.D., M.P.H.

Director, Task Order Officer

Evidence-based Practice Center Program

Center for Evidence and Practice Improvement Agency for Healthcare Research and Quality
Arlene Bierman, M.D., M.S.

Director

Center for Evidence and Practice

Improvement

Agency for Healthcare Research and Quality

Diana Pham, M.S.S.W.

Task Order Officer

Center for Evidence and Practice

Improvement

Agency for Healthcare Research and Quality 


\section{Acknowledgments}

The authors gratefully acknowledge the following individuals for their contributions to this project: Shana Ratner; Julia Tompkins; Bailey Minish; Garrett Thompson; Nancy McElveen; and Laura Brown and the staff of the Institute for Healthcare Quality Improvement.

\section{Key Informants}

In designing the study questions, the EPC consulted several Key Informants who represent the end-users of research. The EPC sought the Key Informant input on the priority areas for research and synthesis. Key Informants are not involved in the analysis of the evidence or the writing of the report. Therefore, in the end, study questions, design, methodological approaches, and/or conclusions do not necessarily represent the views of individual Key Informants.

Key Informants must disclose any financial conflicts of interest greater than $\$ 5,000$ and any other relevant business or professional conflicts of interest. Because of their role as end-users, individuals with potential conflicts may be retained. The TOO and the EPC work to balance, manage, or mitigate any conflicts of interest.

The list of Key Informants who provided input to this report follows:

Andrew Felcher, M.D.

Kaiser Permanente NW

Clackamas, OR

Kenneth Lin, M.D.

Department of Family Medicine, Georgetown University

Washington, DC

Shana Ratner, M.D.

General Internal Medicine, University of North Carolina at Chapel Hill

Chapel Hill, NC

Julia Tompkins, M.S.W., L.C.S.W.

General Internal Medicine, University of North Carolina at Chapel Hill

Chapel Hill, NC

Courtney Wolk, Ph.D.

Perelman School of Medicine, University of Pennsylvania

Philadelphia, PA

Judith Zerzan, M.D., M.P.H.

Colorado Department of Health Care Policy and Financing

Denver, CO 


\section{Development of a Primary Care Guide for Implementing Evidence-based Screening and Counseling for Unhealthy Alcohol Use with Epic- based Electronic Health Record Tools: A Pilot Dissemination Project}

\section{Structured Abstract}

Background. Based on an Evidence-based Practice Center (EPC) systematic review, the U.S. Preventive Services Task Force (USPSTF) recommended that clinicians screen adults aged 18 years or older for unhealthy alcohol use and provide counseling for persons engaged in risky drinking. Relying on the EPC review, we implemented an evidence-based, systematic approach to screening and counseling in a General Internal Medicine (GIM) Clinic beginning in July 2016. The approach included Epic electronic health record tools, including visit-based reminders for nurses and providers. In the current methods pilot project, we aimed to (1) further evaluate the implementation and (2) to produce a dissemination package for use by other health systems that describes our implementation and quality improvement process and offers actionable steps to assist other clinics or health care systems wishing to implement similar evidence-based practices.

Methods. We used multiple sources of information to evaluate our implementation and produce a dissemination package (separate document). ${ }^{1}$ Evaluation of the initial implementation and quality improvement project included collection of data and creation of run charts to track outcome measures for the following: number and percentage of patients screened from the clinic population, fidelity to the screening protocol, and proportion of patients offered counseling for risky drinking, when indicated. Additionally, to assess lessons learned, challenges, and barriers, we conducted semi-structured interviews, guided by the RE-AIM (Reach, Efficacy, Adoption, Implementation, Maintenance) framework, with members of the original implementation project team. Representatives of health systems served as key informants (KIs) to provide an evaluation of the draft dissemination package. Feedback from an online questionnaire completed by the KIs was used to revise the package.

Results. Data collection and tracking for the initial implementation and quality improvement project showed that over 9,000 patients (>70\% of eligible patients) were screened over 18 months and 64 percent of patients who had positive initial screens had documented screeningrelated assessment with the Alcohol Use Disorders Identification Test. Forty percent (141 of 355 ) were offered counseling for risky drinking when indicated. These results compare very favorably to national rates as fewer than 25 percent of U.S. adults report ever discussing alcohol use with a health professional, fewer than half receive any follow up after identification of unhealthy alcohol use, and fewer than 25 percent of those with significant problems from alcohol use receive a recommendation to stop drinking. Interviews with implementation project team members suggested that EHR tools facilitated provision of the service but might contribute to 
alert fatigue. Other facilitators included clinic leadership support; a culture of innovation and continuous QI; a strong multidisciplinary team; and, an organized plan for training faculty and residents. Clinic and visit factors such as competing demands, a large patient population, and turnover in nursing staff and resident providers were identified as potential barriers. The dissemination package contains components corresponding to the most important aspects of the implementation process, with descriptions specific to screening and counseling for unhealthy alcohol use (including resources such as screening instruments, and patient and provider materials to support counseling) as well as more general guidance for implementing evidencebased services in primary care. Six KIs completed the package review and evaluation; most (10/12) of the product sections were rated as very useful by a majority of the KIs. Specifically, all KIs found components of the package that provided direct and practical guidance for implementation to be very useful; components dealing with more general aspects of implementation were also rated as very useful by most but were considered somewhat useful or not particularly useful by some. Suggestions for improvement focused on issues of clarity and organization, as well as making the package less Epic-specific to allow for broader applicability.

Conclusions. Evidence-based screening and counseling for unhealthy alcohol use can be successfully implemented with electronic health record tools; our initial quality improvement project, which benefited from dedicated funding and a multi-disciplinary team, resulted in screening and counseling rates greater than those reported in national data. A dissemination package describing the process, barriers, and facilitators was viewed favorably by KIs. Similar dissemination packages could be developed for other EPC reports, but to be feasible additions to the scope of work would require expansion of the timeline and resources as well as input from recent implementation of the evidence-based service.

1. Barclay C, Viswanathan M, Ratner SP, et al. Implementing Evidence-based Screening and Counseling for Unhealthy Alcohol Use with Epic-based Electronic Health Record Tools: A Guide for Clinics and Health Systems, Developed as Part of a Pilot Dissemination Project (Prepared by the RTI International-University of North Carolina Evidence-based Practice Center under Contract No.290-2015-00011-I.) AHRQ Publication No. 18-EHC020-1-EF. Rockville, MD: Agency for Healthcare Research and Quality; September 2018. DOI: https://doi.org/10.23970/AHRQEPCMETHENGAGEALCOHOLGUIDE. Posted final reports are located on the Effective Health Care Program search page. 


\section{Contents}

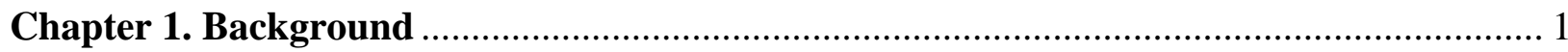

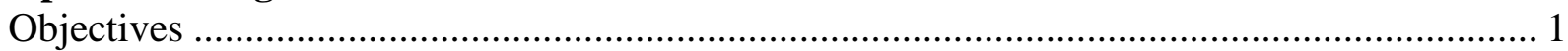

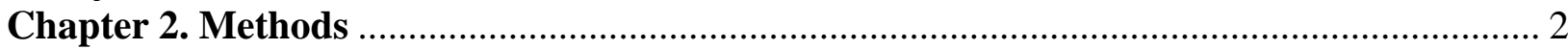

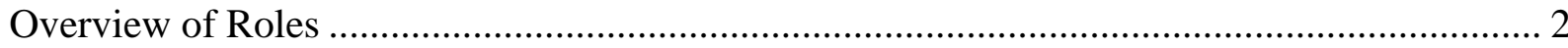

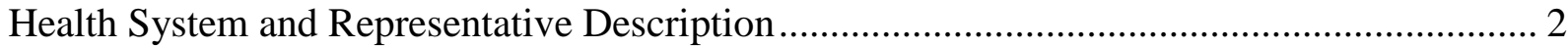

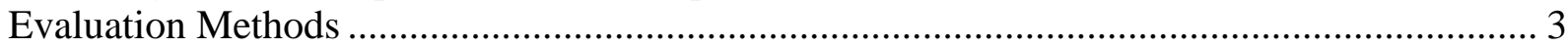

Process Description: Development and Revision of Dissemination Package ........................... 3

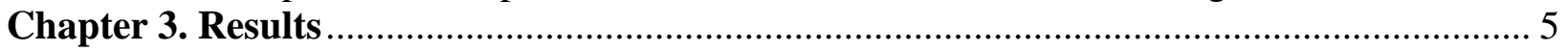

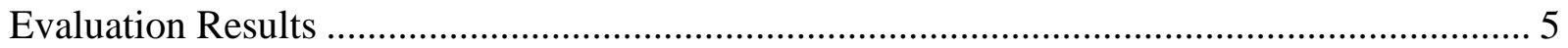

Initial Implementation and Quality Improvement Project............................................. 5

Feedback from Original Implementation Team Member Interviews ................................. 7

Development of the Dissemination Package .................................................................. 9

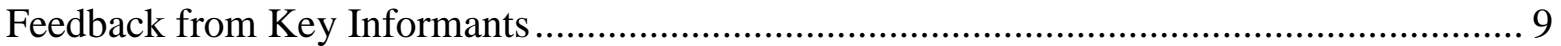

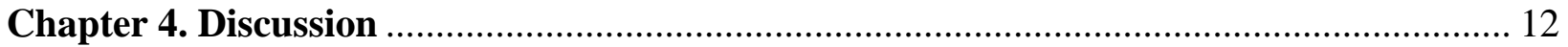

Utility and Applicability for Other Health Systems .......................................................... 12

Lessons Learned and Applicability for Other EPC Reports .............................................. 12

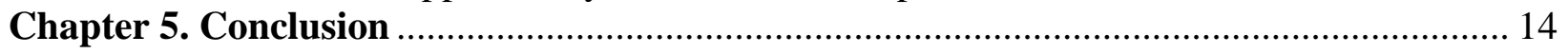

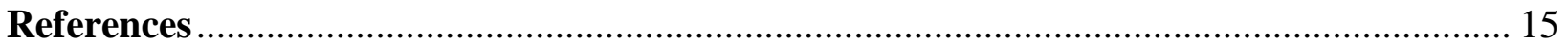

Exhibits

Exhibit 1. Outline for dissemination package.................................................................. 9

Tables

Table 1. Participants in implementation, evaluation, and package development ......................... 2

Table 2.Implementation team interviews: Results organized by question dimension and response

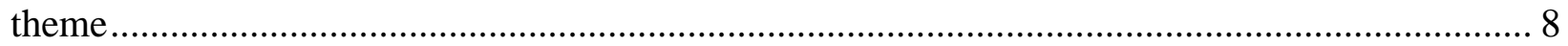

Table 3. Key informant ratings of dissemination package sections........................................ 10

Table 4. Summary of suggested improvements ............................................................... 10

\section{Figures}

Figure 1. Total patients screened for unhealthy alcohol use ................................................. 6

Figure 2. Proportion of eligible patients visits in which initial screen for unhealthy alcohol use was completed........................................................................................................... 6

Figure 3. Proportion of patients with positive initial screen who had AUDIT results documented7 Figure 4. Patients offered counseling for risky drinking when indicated on day of screening....... 7

\section{Appendixes}

Appendix A. Interview Questions for Original Team Members

Appendix B. Questionnaire for Key Informants 


\section{Chapter 1. Background}

Evidence reviews by EPCs are used by groups, such as clinical professional organizations, healthcare organizations, and Federal agencies, to inform clinical practice guideline development, program planning, and research priorities. The AHRQ EPC program aims to optimize the utility and uptake of existing EPC reports by learning health systems. To support this goal, the EPCs engaged health systems to develop and test dissemination products that will help health systems use EPC reports.

The RTI International-University of North Carolina Evidence-based Practice Center produced a systematic review on screening, behavioral counseling, and referral in primary care to reduce unhealthy alcohol use. ${ }^{1,2}$ The report was used by the U.S. Preventive Services Task Force (USPSTF) to recommend (in 2013) that clinicians screen adults aged 18 years or older and provide persons engaged in risky drinking with brief behavioral counseling interventions. ${ }^{3}$

Unhealthy alcohol use is the third leading cause of preventable deaths among working-age adults in the US. ${ }^{4}$ Despite the burden of illness and recommendations to screen for unhealthy alcohol use, the local health care system lacked a formal process for screening and subsequent delivery of appropriate interventions for primary care patients. Our healthcare system implemented a new electronic health record (Epic) in 2014, providing an opportunity to implement a new process utilizing electronic tools and reminders, following the establishment of some other evidence-based practices that made use of such tools.

In our initial implementation and quality improvement project we aimed to fill gaps in the delivery of care for primary care patients with unhealthy alcohol use by: (1) implementing and evaluating an evidence-based, systematic approach to screening in the General Internal Medicine Clinic; (2) training resident and faculty providers in evidence-based screening and counseling for unhealthy alcohol use, and 3) using PDSA cycles (Plan, Do, Study, Act; a quality improvement method $^{5}$ ) with low tech approaches to build visit-based reminders (also called Best Practice Advisories [BPAs]) for nurses and providers that facilitate screening and appropriate interventions and referrals.

\section{Objectives}

1. To further evaluate the evidence-based approach to screening and offering interventions for unhealthy alcohol use that we recently implemented (based on the EPC evidence report we produced), and to summarize lessons learned.

2. To produce a package (separate document, titled Implementing Evidence-based Screening and Counseling for Unhealthy Alcohol Use with Epic-based Electronic Health Record Tools: A Guide for Clinics and Health Systems ${ }^{6}$ ) that can be used by other health systems, describing how we implemented findings from a systematic review (an EPC report) on alcohol screening and counseling, and offer a list of actionable steps to assist other clinics or healthcare systems (or other EPCs) that are in the early stages of disseminating and implementing similar evidence-based practices. 


\section{Chapter 2. Methods}

\section{Overview of Roles}

Table 1 summarizes the roles of the individuals involved in the original implementation project and its evaluation, as well as the key informants who provided feedback during the development of the dissemination package. Some participants filled multiple roles.

Table 1. Participants in implementation, evaluation, and package development

\begin{tabular}{|l|c|c|c|}
\hline Name of participant & $\begin{array}{c}\text { Implementation } \\
\text { Team Member }\end{array}$ & Key Informant & $\begin{array}{c}\text { Health System } \\
\text { Representative }\end{array}$ \\
\hline Dan Jonas & $\mathrm{X}$ & $\mathrm{X}$ & $\mathrm{X}$ \\
\hline Shana Ratner & $\mathrm{X}$ & $\mathrm{X}$ & $\mathrm{X}$ \\
\hline Julia Tompkins & $\mathrm{X}$ & & \\
\hline Garrett Thompson & $\mathrm{X}$ & $\mathrm{X}$ & $\mathrm{X}$ \\
\hline Nancy McElveen & $\mathrm{X}$ & $\mathrm{X}$ & $\mathrm{X}$ \\
\hline Andrew Felcher & & $\mathrm{X}$ & $\mathrm{X}$ \\
\hline Kenneth Lin & & $\mathrm{X}$ & $\mathrm{X}$ \\
\hline Courtney Wolk & & & \\
\hline Judith Zerzan & & & \\
\hline
\end{tabular}

${ }^{a}$ Interviewed for evaluation of the original implementation project. ${ }^{b}$ Provided feedback on dissemination package

\section{Health System and Representative Description}

The original implementation of screening and counseling for unhealthy alcohol use was conducted at the University of North Carolina (UNC) General Internal Medicine (GIM) Clinic, a large academic practice serving over 12,000 adults with approximately 42,000 visits per year. ${ }^{7}$ Recognized as a Level 3 Patient-Centered Medical Home, the GIM Clinic provides a team approach to primary and consultative clinical care to both the inpatient and outpatient population at UNC Hospitals and affiliated practices. Three of the original team members, described below, served as health system representatives in the evaluation of the implementation/quality control project.

Shana Ratner, MD, is the Clinic Director for the GIM Clinic and was the primary health system representative. Dr. Ratner has expertise in practice innovation and quality improvement, including multiple Lean Six Sigma Certifications from her Medical Center's Department of Operational Efficiency. She served as project coach and sponsor for the initial implementation and quality improvement effort. Dr. Ratner was interviewed as a member of the original team, and also served as a key informant, reviewing the package developed during the current project and providing feedback via an online questionnaire.

Julia Tompkins, MSW, LCSW-A, is the GIM Clinic social worker involved in the initial implementation. During that process, she led the development of a comprehensive list of local resources, by county and type, for patients with unhealthy alcohol use. Ms. Tompkins was interviewed as a member of the original team, and also served as a key informant, reviewing the package developed during the current project and providing feedback via an online questionnaire.

Daniel E. Jonas, MD, MPH, is an experienced clinician-investigator with expertise in unhealthy alcohol use and Co-Director of the RTI-UNC Evidence-based Practice Center. Dr. Jonas led the implementation and quality improvement efforts and is directing the current project. 
Four additional individuals not part of the original implementation team served as key informants who contributed feedback on the draft dissemination package, as described in the following subsection. They are Andrew Felcher, MD (Director, NWP Guidelines, EvidenceBased Medicine and Shared Decision Making at Kaiser Permanente NW); Gillian Lautenbach (Associate Chief, Ambulatory, Division of General Internal Medicine at University of Pennsylvania); Kenneth Lin, MD (Associate Professor of Family Medicine at Georgetown University); Courtney Wolk, PhD (Postdoctoral Researcher, Perelman School of Medicine at University of Pennsylvania); and Judith Zerzan, MD, MPH (Chief Medical Officer and Client and Clinical Care Office Director, Colorado Department of Health Care Policy and Financing).

\section{Evaluation Methods}

Evaluation of the initial implementation and quality improvement project included collection of data and creation of run charts to track the following measures:

- Total number (and percent) of patients screened from the clinic population

- Fidelity to screening protocol (proportion of eligible patients screened each month, proportion of patients who had positive initial screen with AUDIT results documented)

- Proportion of patients offered counseling for risky drinking, when indicated based on screening results and screening-related assessment (and based on documentation in the electronic health record)

To assess lessons learned, challenges, and barriers—-for example, related to creating buy-in from providers and nursing staff, time burden, information needed, incorporating patient perspectives, and engaging health systems - we conducted semi-structured interviews with the original team members, incorporating questions based on dimensions described in the RE-AIM (Reach, Efficacy, Adoption, Implementation, Maintenance) framework ${ }^{8}$ (Appendix A). In addition to the health system representatives on the original team, described above, other original team members who participated in the evaluation are Bailey Minish (research assistant), Garrett Thompson (Improvement Scholar), and Nancy McElveen (patient representative).

\section{Process Description: Development and Revision of Dissemination Package}

Drawing upon our experiences implementing a formal process for screening and subsequent delivery of appropriate interventions for unhealthy alcohol use among primary care patients at the GIM Clinic, we identified the most important components from the initial implementation and quality improvement project period. One team member (Colleen Barclay) reviewed project files, including forms documenting the PDSA cycles and the step-by-step screening and counseling clinic protocols, and recorded relevant details about development and testing of protocols and tools. In sections of the package corresponding to the most important components, we described our implementation process as well as offering more general guidance related to implementation of evidence-based services in primary care settings. Examples of process diagrams and screening tools were included. The package includes descriptions of tools that may accelerate dissemination, such as 3 Epic-based visit-based reminders, and lessons learned (including challenges and barriers). Two team members (CB and Dan Jonas) produced the draft package and revised it after review by the third member (Meera Viswanathan) and by AHRQ staff. 
To evaluate the dissemination package, we identified and solicited feedback from key informants (KIs). We conducted an initial online search for representatives of healthcare systems using Epic EHR (both academic and non-academic, of various sizes and from various regions of the United States), such as clinic medical directors, in a position to make decisions about implementation of services into primary care. Finding that contact information for such individuals was often unavailable on healthcare system web pages, we proceeded with a snowball sampling strategy, ${ }^{9}$ contacting General Internal Medicine division heads, asking our TOO on this project to suggest colleagues, and contacting directors at other EPCs with a brief explanation of the project and a request to recommend appropriate individuals who might serve as KIs.

Individuals who agreed to serve as KIs were asked to review the draft package and provide input, via individualized links to a Qualtrics questionnaire (Appendix B), focusing on which components were useful and which were not useful, as well as general feedback on the package. Additionally, we sought information that may help other clinics or health systems with uptake and factors that may predict successful dissemination and implementation.

Where feedback from the key informants' evaluation of the revised draft clearly indicated ways in which the package could be improved, we modified the document according to their suggestions. 


\section{Chapter 3. Results}

\section{Evaluation Results}

\section{Initial Implementation and Quality Improvement Project}

Our initial project took place, with quality improvement initiative funding, over the course of a year, during which a multidisciplinary team met regularly to refine processes. During the project period, time dedicated by team members varied according to role: the Project Lead and Clinic Medical Director each contributed approximately 120 hours; the Project Coordinator and Clinic Project Assistant's combined effort was about 1,000 hours; and work by other personnel (Nurse Manager, Social Worker, Patient Representative, and a student QI participant) totaled another 90-100 hours. Run charts of data over time for 4 measures are shown in Figures 1-4. The project team collected these data and created run charts at regular intervals to monitor progress while alcohol screening and counseling were being incorporated into clinic workflow, to help us identify inflection points corresponding to introduction of new processes, to assess for changes after modifying processes, and to evaluate the success of the implementation. We have continued to collect data since the end of the project period in order to assess maintenance.

A systematic process for screening for unhealthy alcohol use was not in place at our site at the outset of this implementation project; therefore, pre-implementation data was not available. Screening for unhealthy alcohol use is being performed at about 64 percent of eligible patient visits. These rates exceed national estimates, in which less than 25 percent of U.S. adults report ever discussing alcohol use with a health professional. ${ }^{10}$ The proportion of our patients with positive screens for unhealthy alcohol use who have been offered counseling (about 40\%) compares favorably with national rates showing that less than 25 percent of those with significant problems from alcohol use received a recommendation to stop drinking ${ }^{11}$ —but falls short of our admittedly lofty target level.

Our data may underestimate the frequency of counseling, since it was collected for day of screening and thus does not capture counseling offered on subsequent visits. In addition to the barriers identified in team member interviews (e.g., competing demands during visits, turnover among resident providers, undertraining of part-time providers, data collection limited to the day of screening), another influence on rates of counseling is worth noting. Individuals with unhealthy drinking patterns who are screened for the first time are often in the pre-contemplative stage of change, as described in the Transtheoretical Model of behavior change. ${ }^{12}$ Although our measures specified intervention offered rather than completed, rates of documentation may be lower when a patient declines counseling. A positive screen may itself conceivably have an effect over time on a patient's readiness to change, even if counseling is not accepted during the encounter itself. 
Figure 1. Total patients screened for unhealthy alcohol use

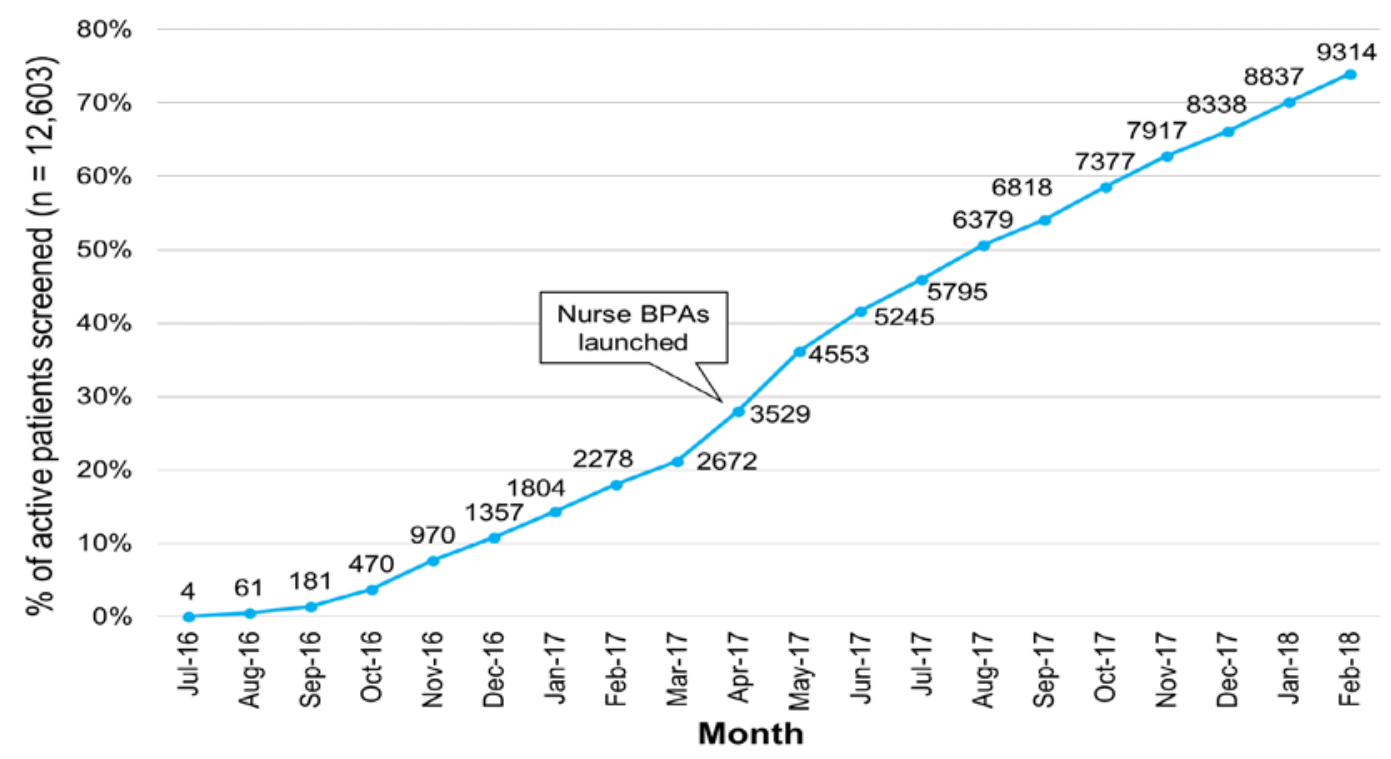

Figure 2. Proportion of eligible patients visits in which initial screen for unhealthy alcohol use was completed

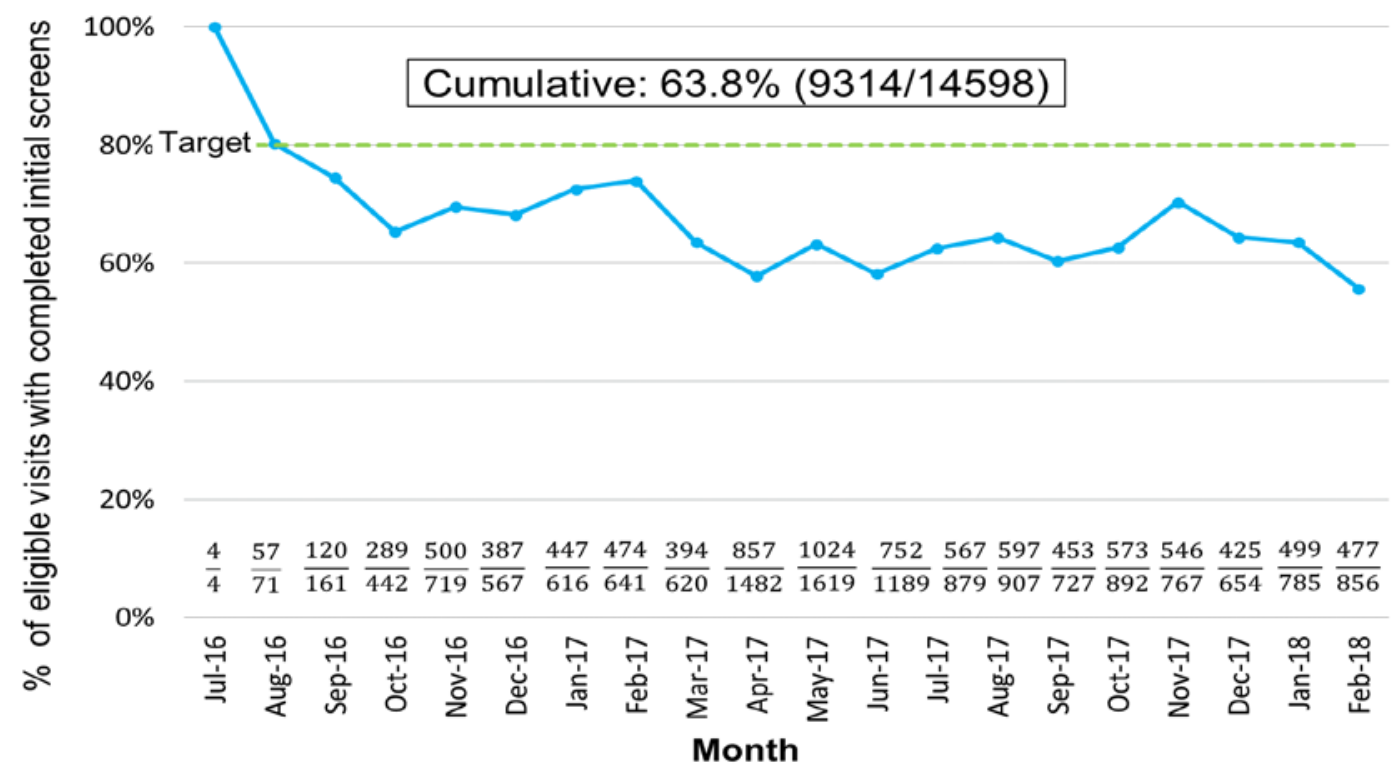


Figure 3. Proportion of patients with positive initial screen who had AUDIT results documented

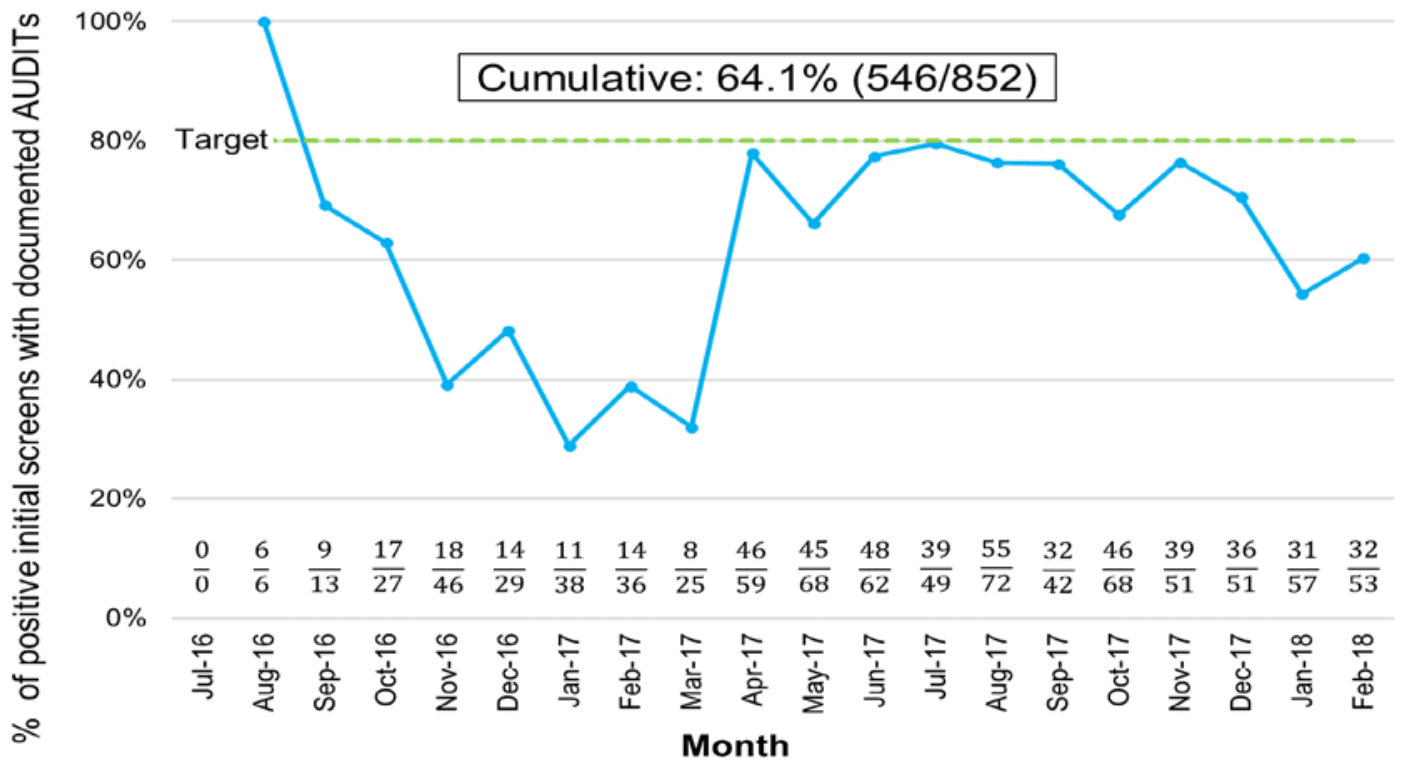

Figure 4. Patients offered counseling for risky drinking when indicated on day of screening

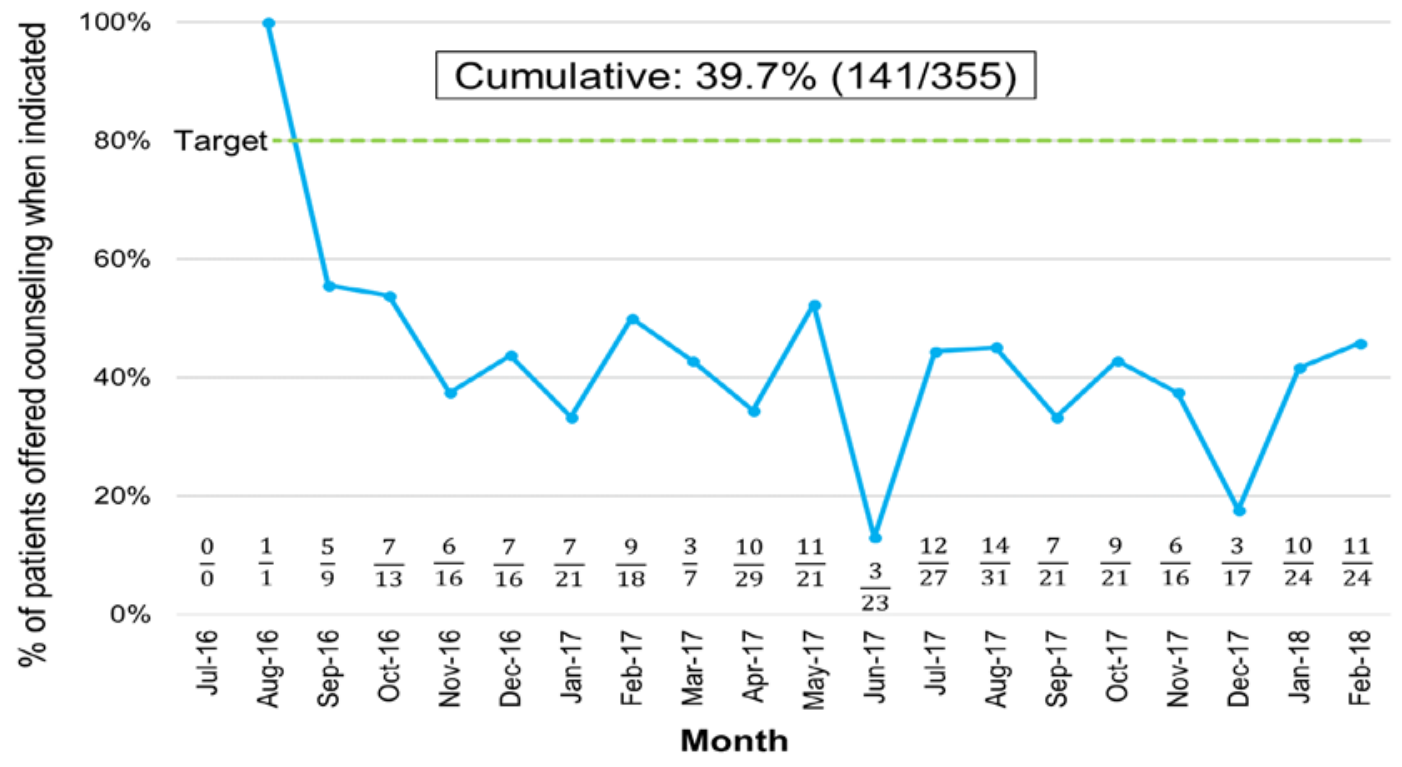

\section{Feedback From Original Implementation Team Member Interviews}

We conducted semi-structured interviews with six members of the original implementation project team. Our questions, based on dimensions described in the RE-AIM (Reach, Efficacy, Adoption, Implementation, Maintenance) framework, ${ }^{8}$ are shown in Appendix A. One investigator (CB) reviewed the interview transcripts and identified themes corresponding to the most important influences on implementation success; these are organized in relation to the framework dimensions and summarized in Table 2. 
Table 2. Implementation team interviews: Results organized by question dimension and response theme

\begin{tabular}{|c|c|c|c|c|c|c|}
\hline Dimension & $\begin{array}{l}\text { Theme: EHR } \\
\text { tools }\end{array}$ & $\begin{array}{l}\text { Theme: } \\
\text { Team } \\
\text { capacity }\end{array}$ & $\begin{array}{l}\text { Theme: Clinic } \\
\text { and visit factors }\end{array}$ & $\begin{array}{l}\text { Theme: } \\
\text { Training and } \\
\text { education }\end{array}$ & $\begin{array}{l}\text { Theme: } \\
\text { Provider } \\
\text { characteristics }\end{array}$ & $\begin{array}{l}\text { Theme: Patient } \\
\text { characteristics }\end{array}$ \\
\hline Facilitators & $\begin{array}{l}\text { BPAs \& } \\
\text { SmartPhrases }\end{array}$ & $\begin{array}{l}\text { Engagement } \\
\text { with nursing } \\
\text { staff; } \\
\text { promotion of } \\
\text { buy-in; } \\
\text { development } \\
\text { of support } \\
\text { materials; use } \\
\text { of QI } \\
\text { methodology }\end{array}$ & $\begin{array}{l}\text { Support from } \\
\text { clinic leadership; } \\
\text { clinic with long } \\
\text { history of } \\
\text { practice } \\
\text { innovation and } \\
\text { continuous QI } \\
\text { culture }\end{array}$ & $\begin{array}{l}\text { Organized plan } \\
\text { for training } \\
\text { faculty and } \\
\text { residents }\end{array}$ & NA & NA \\
\hline Barriers & Alert fatigue & NA & $\begin{array}{l}\text { Competing } \\
\text { demands in visit; } \\
\text { large patient } \\
\text { population; part- } \\
\text { time providers; } \\
\text { resident \& nurse } \\
\text { turnover; float } \\
\text { nurses }\end{array}$ & $\begin{array}{l}\text { Lack of provider } \\
\text { familiarity with } \\
\text { counseling }\end{array}$ & NA & NA \\
\hline Reach & NA & NA & $\begin{array}{l}\text { Competing } \\
\text { priorities in clinic; } \\
\text { intervention and } \\
\text { referral } \\
\text { resources }\end{array}$ & $\begin{array}{l}\text { Patient } \\
\text { perspectives } \\
\text { helped develop } \\
\text { patient } \\
\text { education } \\
\text { materials; }\end{array}$ & $\begin{array}{l}\text { Lack of } \\
\text { continuity with } \\
\text { patients }\end{array}$ & $\begin{array}{l}\text { Visit frequency; } \\
\text { age; AUDIT } \\
\text { score }\end{array}$ \\
\hline Effectiveness & Alert fatigue & $\begin{array}{l}\text { Development } \\
\text { of materials }\end{array}$ & $\begin{array}{l}\text { Competing } \\
\text { demands; } \\
\text { resident turnover }\end{array}$ & $\begin{array}{l}\text { Enhanced } \\
\text { knowledge of } \\
\text { intervention and } \\
\text { referral } \\
\text { resources }\end{array}$ & NA & $\begin{array}{l}\text { Opportunities for } \\
\text { patient education }\end{array}$ \\
\hline Adoption & $\begin{array}{l}\text { Provider } \\
\text { familiarity with } \\
\text { tools }\end{array}$ & NA & $\begin{array}{l}\text { Float nurses; } \\
\text { some low- } \\
\text { performing } \\
\text { nurses }\end{array}$ & NA & $\begin{array}{l}\text { Attendance at } \\
\text { division } \\
\text { meetings and } \\
\text { participation in } \\
\text { discussions; } \\
\text { proximity to } \\
\text { project }\end{array}$ & NA \\
\hline Maintenance & BPAs & $\begin{array}{l}\text { Manual data } \\
\text { entry of } \\
\text { AUDITs }\end{array}$ & $\begin{array}{l}\text { Resident } \\
\text { turnover }\end{array}$ & $\begin{array}{l}\text { Ongoing } \\
\text { training }\end{array}$ & NA & NA \\
\hline $\begin{array}{l}\text { Challenge } \\
\text { encountered }\end{array}$ & $\begin{array}{l}\text { BPA } \\
\text { functionality }\end{array}$ & $\begin{array}{l}\text { New skills } \\
\text { acquisition; } \\
\text { challenges } \\
\text { addressed } \\
\text { with PDSAs }\end{array}$ & $\begin{array}{l}\text { Patient backlog } \\
\text { for screening }\end{array}$ & NA & NA & NA \\
\hline $\begin{array}{l}\text { Implementation } \\
\text { at other sites }\end{array}$ & $\begin{array}{l}\text { BPAs \& } \\
\text { SmartPhrases; } \\
\text { standardized } \\
\text { documentation } \\
\text { processes }\end{array}$ & $\begin{array}{l}\text { Staff \& } \\
\text { provider buy- } \\
\text { in; } \\
\text { development } \\
\text { of materials; } \\
\text { ongoing } \\
\text { training; } \\
\text { follow-up of } \\
\text { AUDITs }\end{array}$ & NA & $\begin{array}{l}\text { Motivational } \\
\text { interview } \\
\text { techniques; } \\
\text { knowledge of } \\
\text { intervention and } \\
\text { referral } \\
\text { resources; use } \\
\text { of EHR tools }\end{array}$ & $\begin{array}{l}\text { Changes in } \\
\text { provider } \\
\text { behavior }\end{array}$ & NA \\
\hline
\end{tabular}


Abbreviation: BPA = Best Practice Advisory (a type of visit-based reminder); QI = quality improvement; AUDIT = Alcohol Use Disorders Identification Test; PDSA = Plan/Do/Study/Act (a QI method involving small tests of change)

\section{Development of the Dissemination Package}

We produced a dissemination package (Implementing Evidence-based Screening and Counseling for Unhealthy Alcohol Use with Epic-based Electronic Health Record Tools: A Guide for Clinics and Health Systems ${ }^{6}$ ) (Exhibit 1). This guide was based on the USPSTF recommendation; ${ }^{3}$ the EPC review on Screening, Behavioral Counseling, and Referral in Primary Care to Reduce Alcohol Misuse, prepared by the RTI-UNC EPC; ${ }^{1,2}$ our prior experiences with and publications on alcohol screening and counseling; ${ }^{13-16}$ and publicly available materials developed by the NIAAA. ${ }^{17}$ The EPC review's authors concluded that available evidence showed that counseling interventions improve behavioral outcomes (e.g., reduction in amount of alcohol consumed, fewer heavy drinking episodes, recommended drinking limits achieved), with the best evidence being for brief multi-contact interventions. Additional materials from the EPC review that were used in the development of the screening and counseling process that we implemented included use of the single-question screen recommended by the National Institute on Alcohol Abuse and Alcoholism (NIAAA), followed by the full 10-question Alcohol Use Disorders Identification Test (AUDIT). ${ }^{17}$

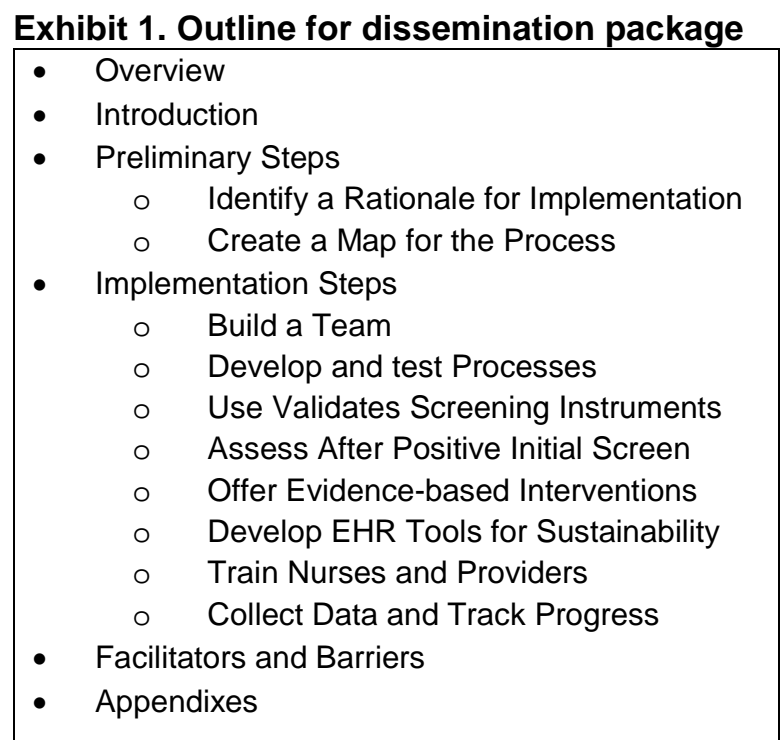

Counseling interventions used techniques from motivational interviewing, an evidence-based behavioral counseling approach that uses a patient-centered, guiding (rather than directing) style to elicit behavior change by helping patients to explore and resolve ambivalence, and identify their personal motivations for change. ${ }^{18,19}$ Provider and patient pamphlets were developed for use in (or to support) counseling and included portions of publicly available materials developed by the NIAAA. ${ }^{17}$

\section{Feedback From Key Informants}

Six KIs reviewed the dissemination package and completed the questionnaire. Ratings of the package sections' usefulness are summarized in Table 2 , and responses to free-text questions about improving the package's usefulness in Table 3. 
Table 3. Key informant ratings of dissemination package sections

\begin{tabular}{|l|c|c|c|c|}
\hline Section & $\begin{array}{l}\text { No. of KIs who } \\
\text { rated section } \\
\text { "very useful" }\end{array}$ & $\begin{array}{l}\text { No. of KIs who } \\
\text { rated section } \\
\text { "somewhat } \\
\text { useful" }\end{array}$ & $\begin{array}{l}\text { No. of KIs who } \\
\text { rated section } \\
\text { "not particularly } \\
\text { useful" }\end{array}$ & $\begin{array}{l}\text { No. of KIs who } \\
\text { rated section } \\
\text { "not useful" }\end{array}$ \\
\hline Overview & 4 & 1 & 1 & 0 \\
\hline Identify a Rationale for Implementation & 5 & 1 & 0 & 0 \\
\hline Create a Map of the Process & 4 & 2 & 0 & 0 \\
\hline Build a Team & 4 & 1 & 1 & 0 \\
\hline Develop and Test Process & 4 & 2 & 0 & 0 \\
\hline Use Validated Screening Instruments & 6 & 0 & 0 & 0 \\
\hline Assess After Positive Initial Screen & 4 & 1 & 1 & 0 \\
\hline Offer Evidence-based Interventions & 5 & 1 & 0 & 0 \\
\hline Develop EHR Tools for Sustainability & 5 & 0 & 1 & 0 \\
\hline Train Nurses and Providers & 4 & 1 & 0 & 0 \\
\hline Collect Data and Track Progress & 6 & 0 & 0 & 0 \\
\hline Barriers and Facilitators & 4 & 1 & 1 & 0 \\
\hline
\end{tabular}

Table 4. Summary of suggested improvements

\begin{tabular}{|c|c|}
\hline Section & Suggestions for improvement \\
\hline Overview & $\begin{array}{l}\text { - } \quad \text { More clarity and consistency in organization } \\
\text { - } \quad \text { Add language after initial paragraphs for transition-what to expect and what the goals were }\end{array}$ \\
\hline $\begin{array}{l}\text { Identify a Rationale } \\
\text { for Implementation }\end{array}$ & $\begin{array}{l}\text { - Look at baseline data } \\
\text { - Specify that counseling is effective; address provider perception that counseling will fail }\end{array}$ \\
\hline $\begin{array}{l}\text { Create a Map of the } \\
\text { Process }\end{array}$ & $\begin{array}{l}\text { - Explain briefly how the process flow diagram and Epic reminders emerged from the evidence } \\
\text { base }\end{array}$ \\
\hline Build a Team & - Be more specific about type/number of staff needed to fill roles \\
\hline $\begin{array}{l}\text { Develop and Test } \\
\text { Process }\end{array}$ & $\begin{array}{l}\text { - Start out everything low-tech } \\
\text { - Don't use Epic terms (instead, terms like "user-editable tools" for things that don't require } \\
\text { EHR build team involvement) }\end{array}$ \\
\hline $\begin{array}{l}\text { Use Validated } \\
\text { Screening } \\
\text { Instruments }\end{array}$ & - \\
\hline $\begin{array}{l}\text { Assess After Positive } \\
\text { Initial Screen }\end{array}$ & $\begin{array}{l}\text { - Advise clinics to get stakeholder feedback when deciding whether to do paper vs flowsheet } \\
\text { type processes, with consideration to fidelity to process. }\end{array}$ \\
\hline $\begin{array}{l}\text { Offer Evidence- } \\
\text { based Interventions }\end{array}$ & $\begin{array}{l}\text { - Provide references for information on performing brief interventions or motivational } \\
\text { interviewing }\end{array}$ \\
\hline $\begin{array}{l}\text { Develop EHR Tools } \\
\text { for Sustainability }\end{array}$ & $\begin{array}{l}\text { - Make less technical/specific; more general discussion of elements } \\
\text { - } \quad \text { Add suggestions for clinics with EHR other than Epic } \\
\text { - } \quad \text { Information on cost, resources needed, institutional buy-in } \\
\end{array}$ \\
\hline $\begin{array}{l}\text { Train Nurses and } \\
\text { Providers }\end{array}$ & - Move this section to follow Offer Evidence-based Interventions \\
\hline $\begin{array}{l}\text { Collect Data and } \\
\text { Track Progress }\end{array}$ & $\begin{array}{l}\text { - Add process measurements (number of times intervention performed; provider satisfaction; } \\
\text { patient satisfaction } \\
\text { - Add outcome measures (number of referrals to alcohol programs; number of patients with } \\
\text { alcohol problem successfully treated; compare to pre-implementation numbers }\end{array}$ \\
\hline $\begin{array}{l}\text { Barriers and } \\
\text { Facilitators }\end{array}$ & - Add targeted implementation strategies for overcoming barriers \\
\hline Overall & $\begin{array}{l}\text { - } \quad \text { Add language in each section that introduces examples } \\
\text { - Add a case scenario where successful implementation was shown to lead to positive } \\
\text { outcomes for the patient } \\
\text { - } \quad \text { Distill information into steps or sequence of activities; or, add a checklist of activities }\end{array}$ \\
\hline
\end{tabular}


KIs were also asked what factors they considered most predictive of successful dissemination and implementation efforts. Responses included:

- Strong evidence base

- Confidence in the practice that implementation won't require too many additional resources

- The presence of practice champions

- Early engagement of all stakeholders and frequent feedback from them

- Leveraging power of story as well as data

- Frequent and early small tests of change

- Transparency of data

- Build in sustainability from the start

- Buy-in from the team

- Organizational culture

- Leadership support for implementation

Feedback from the KIs' evaluation of the revised draft has been incorporated into the dissemination package. 


\section{Chapter 4. Discussion}

\section{Utility and Applicability for Other Health Systems}

Evaluation of the draft package by six key informants indicated that it provides helpful guidance for other health systems wishing to implement screening and counseling for unhealthy alcohol use in primary care clinics. The 12 sections of the package, each corresponding to an important component of the implementation process we developed and tested in our initial quality improvement project, were rated as "very useful” by a majority of the KIs. All six KIs considered the sections Use Validated Instruments and Collect Data and Track Progress to be very useful, while 5/6 found 3 other sections (Identify a Rationale for Implementation, Offer Evidence-based Interventions, and Develop EHR Tools for Sustainability) to be very useful.

These responses suggest that information with a direct and practical application to the topic of alcohol screening and intervention, and to appropriate metrics for it, was most valued by the KIs. Sections dealing with more general aspects of implementation, (e.g., Create a Map of the Process, Build a Team, Develop and Test Process, Train Nurses and Providers, and Barriers and Facilitators) were rated as very useful by most KIs (4/6) but some rated them as either "somewhat useful" or "not particularly useful."

These responses suggest that end users may want a concise document with a "just tell me what to do" perspective; during our original implementation we received this feedback from any providers. This type of document is also desirable since the level of effort involved in our process (which was conducted with dedicated funding and a multi-disciplinary team) may not be replicable in many systems interested in implementing an evidence-based service.

No KI rated any section as "not useful”. One KI asked for permission to use the package as a model for other work that they are conducting.

Suggestions from KIs about how to improve the package focused on issues of clarity and organization within the document, and the final package will incorporate these as appropriate. Other suggestions for improvement emphasized the importance of a strong evidence base and stakeholder involvement, which are arguably the predictors of success most applicable to implementation efforts in general. And although our KIs were recruited from health systems with Epic EHRs, some suggested reducing the technicality of the material on developing EHR tools and making the terminology less Epic-specific, which could increase applicability for a broader set of health systems Any EHR functionality that included e-reminders is likely to allow development of tools similar to those described in the dissemination package.

\section{Lessons Learned and Applicability for Other EPC Reports}

From team member interviews we identified six themes-EHR tools, team capacity, clinic and visit factors, training \& education, provider characteristics, and patient characteristics-as facilitators, barriers, or influences on RE-AIM dimensions during implementation. Some factors important to implementation at other sites pertained specifically to alcohol screening and interventions (e.g., provider training in counseling methods; resources for intervention and referral) and others were generally applicable (e.g., staff and provider buy-in) to any implementation effort. The importance of practice champions, a supportive organizational culture, openly addressing facilitators and barriers, and leadership support was also noted. These elements have been recognized as components of implementation and sustainability models. ${ }^{18}$ 
A comprehensive high-quality toolkit is often developed over the course of multi-year projects, with extensive evaluation. If packages similar to ours are undertaken for other EPC reports, the creation of concise support tools that do not introduce excessive background information into the busy primary care environment might be both most feasible and most useful and would be facilitated by working with a team that has recently implemented the clinical service in question. Development of the dissemination package for the current pilot project was preceded by nearly two years in which we worked on implementation in the clinical setting. 


\section{Chapter 5. Conclusion}

Evidence-based screening and counseling for unhealthy alcohol use can be successfully implemented with Epic-based electronic health record tools; our initial quality improvement project, which benefited from dedicated funding and a multi-disciplinary team, resulted in screening and evidence-based counseling rates greater than those reported in national data. A dissemination package describing the process, barriers, and facilitators was viewed favorably by key informants. Similar dissemination packages could be developed for other EPC reports but to be feasible additions to the scope of work would require expansion of the timeline and resources as well as input from recent implementation of the evidence-based service. 


\section{References}

1. Jonas DE, Garbutt JC, Amick HR, et al. Behavioral counseling after screening for alcohol misuse in primary care: a systematic review and meta-analysis for the U.S. Preventive Services Task Force. Ann Intern Med. 2012 Nov 6;157(9):645-54. PMID: 23007881. DOI: 10.7326/0003-4819-157-9201211060-00544

2. Jonas DE, Garbutt JC, Brown JM, et al. Screening, Behavioral Counseling, and Referral in Primary Care to Reduce Alcohol Misuse. Comparative Effectiveness Review No. 64. (Prepared by the RTI InternationalUniversity of North Carolina Evidencebased Practice Center under Contract No. 290200710056 I). AHRQ Publication No. 12-EHC055-EF. . Rockville, MD: Agency for Healthcare Research and Quality; 2012.

3. Moyer VA, Preventive Services Task Force. Screening and behavioral counseling interventions in primary care to reduce alcohol misuse: U.S. preventive services task force recommendation statement. Ann Intern Med. 2013 Aug 6;159(3):210-8. PMID: 23698791. DOI: 10.7326/00034819-159-3-201308060-00652

4. Mokdad AH, Marks JS, Stroup DF, et al. Actual causes of death in the United States, 2000. Jama. 2004 Mar 10;291(10):1238-45. PMID: 15010446. DOI:

10.1001/jama.291.10.1238

5. Institute for Healthcare Improvement. Science of Improvement: Testing Changes. 2018.

http://www.ihi.org/resources/Pages/HowtoI mprove/ScienceofImprovementTestingChan ges.aspx. Accessed on May 162018.

6. Barclay C, Viswanathan M, Jonas DE. Implementing Evidence-based Screening and Counseling for Unhealthy Alcohol Use with Epic-based Electronic Health Record Tools: A Guide for Clinics and Health Systems, Developed as Part of a Pilot Dissemination Project. (Prepared by the RTI International-University of North Carolina Evidence-based Practice Center under Contract No. HHSA290201500011I.) AHRQ Publication No. 18-EHC020-1-EF. Rockville, MD: Agency for Healthcare Research and Quality; 2018.
7. School of Medicine. Division of General Medicine \& Clinical Epidemiology. Chapel Hill, NC: University of North Carolina; 2018. http://www.med.unc.edu/im. Accessed on May 182018.

8. Glasgow RE, Vogt TM, Boles SM. Evaluating the public health impact of health promotion interventions: the RE-AIM framework. Am J Public Health. 1999 Sep;89(9):1322-7. PMID: 10474547.

9. Snowball Sampling. In: Lewis-Beck MS, Bryman A, Futing Liao T, eds. The SAGE Encyclopedia of Social Science Research Methods. Thousand Oaks, CA: SAGE Publications; 2004.

10. McKnight-Eily LR, Liu Y, Brewer RD, et al. Vital Signs: Communication Between Health Professionals and Their Patients About Alcohol Use - 44 States and the District of Columbia, 2011. MMWR Morb Mortal Wkly Rep. 2014;63(01):16-22. PMID: 24402468.

11. D'Amico EJ, Paddock SM, Burnam A, et al. Identification of and guidance for problem drinking by general medical providers: results from a national survey. Med Care. 2005 Mar;43(3):229-36. PMID: 15725979.

12. Prochaska JO, Velicer WF. The transtheoretical model of health behavior change. Am J Health Promot. 1997 SepOct;12(1):38-48. PMID: 10170434. DOI: 10.4278/0890-1171-12.1.38

13. Jonas DE, Amick HR, Feltner C, et al. Pharmacotherapy for Adults With AlcoholUse Disorders in Outpatient Settings. Comparative Effectiveness Review No. 134. Rockville MD: Agency for Healthcare Research and Quality; May 2014.

14. Jonas DE, Garbutt JC. Screening and Counseling for Unhealthy Alcohol Use in Primary Care Settings. Med Clin North Am. 2017 Jul;101(4):823-37. PMID: 28577629. DOI: 10.1016/j.mcna.2017.03.011

15. Jonas DE, Garza D. An evidence-based approach to screening and providing appropriate interventions for unhealthy alcohol use in primary care settings. J Comp Eff Res. 2016 Nov;5(6):521-4. PMID: 27582343. DOI: 10.2217/cer-2016-0047 
16. Jonas DE, Miller T, Ratner S, et al. Implementation and quality improvement of a screening and counseling program for unhealthy alcohol use in an academic general internal medicine practice. J Healthc Qual. 2017 Jan/Feb;39(1):15-27. PMID: 28045764. DOI:

10.1097/jhq.0000000000000069
17. National Institute on Alcohol Abuse and Alcoholism. Helping Patients Who Drink Too Much: A Clinician's Guide. Rockville, MD; Updated 2005 ed.

http://pubs.niaaa.nih.gov/publications/Practit ioner/CliniciansGuide2005/guide.pdf. Accessed on March 42015.

18. Feldstein AC, Glasgow RE. A practical, robust implementation and sustainability model (PRISM) for integrating research findings into practice. Jt Comm J Qual Patient Saf. 2008 Apr;34(4):228-43. PMID: 18468362. 


\section{Appendix A. Interview Questions for Original Team Members}

Thank you for taking the time to answer some questions about your experience with the alcohol screening project. We mainly want to get information about lessons learned, challenges, and barriers to the implementation process.

1. What was your role on the alcohol screening implementation team?

2. What factors were facilitators to implementing alcohol use screening and intervention (i.e. helped in delivering the screening and interventions as intended)?

3. What factors were barriers/obstacles to implementing alcohol use screening and intervention (i.e. made it more difficult to deliver the screening and interventions as intended)?

4. Did you observe patterns in or differences among in the patients we were able to reach? What factors do you think explain our reach? What factors prevented us from reaching a wider range of participants Were there other barriers or facilitators to expanding the reach of screening and interventions that you think are important?

5. Do you think the screening and counseling interventions are effective? What do you think helped the screening and interventions be effective? What do you think hindered their effectiveness?

6. Did you observe variations in adoption of the screening and interventions among the nursing staff and providers? What helped them adopt them? What hindered their adoption?

7. What is your experience with maintaining the alcohol screening and interventions? What factors help maintain them? What factors hinder maintenance?

8. Can you tell me about a challenge you faced during the implementation?

a. What was the outcome of this challenge? Do you feel that it was resolved?

9. If we were to implement this package elsewhere, what are the most important issues to keep in mind?

10. Is there anything else you'd like to share about your experience or observations of the implementation process? 


\section{Appendix B. Questionnaire for Key Informants}

1. Please rate the usefulness of each of the sections below, on a scale of 1 to 4 , where $1=$ very useful, 2 = somewhat useful, 3 = not particularly useful, and $4=$ not useful.

- Overview

- Identify a Rationale for Implementation

- Create a Map of the Process

- Build a Team

- Develop and Test Process

- Use Validated Screening Instruments

- Assess after Positive Initial Screen

- Offer Evidence-based Interventions

- Develop EHR Tools for Sustainability

- Train Nurses and Providers

- Collect Data and Track Progress

- Barriers and Facilitators

2. Do you have any suggestions for improving the package?

3. What additional information would help clinics or health systems successfully implement this package?

4. What factors do you consider most predictive of successful dissemination and implementation of this package? 